\title{
Exercício físico regular e depressão em idosos
}

\section{Regular physical exercise and depression in the elderly}

\author{
Ejercicio Físico Regular y Depresión en Ancianos
}

\author{
José Augusto Evangelho Hernandez* \\ Universidade do Estado do Rio de Janeiro - UERJ, Rio de Janeiro, Rio de Janeiro, \\ Brasil
}

\section{Rogério da Cunha Voser**}

Universidade Federal do Rio Grande do Sul - UFRGS, Porto Alegre, Rio Grande do Sul, Brasil

\begin{abstract}
RESUMO
$\mathrm{Na}$ velhice, a capacidade aeróbica, a força muscular, a flexibilidade, o equilíbrio, o tempo de reação e movimento, a agilidade e a coordenação são gradativamente reduzidos. Isso pode acarretar inúmeras dificuldades para a realização das tarefas cotidianas e para a independência funcional dessas pessoas. Esses idosos sentem-se, com frequência, inúteis, ansiosos, frustrados, mal-humorados, irritados e, até, deprimidos. Por outro lado, a prática de atividades físicas regulares pode trazer melhorias para a saúde física e mental dos praticantes. Esta pesquisa investigou a relação entre o exercício físico regular e a depressão em indivíduos idosos. Participaram da pesquisa 160 pessoas de 60 a 85 anos de idade, sendo 84 praticantes de tipos variados de atividades físicas regulares e 76 sedentários. Para avaliar a depressão foi usada Escala de Depressão para Idosos, constituída pelas dimensões cognitiva-afetiva e somática-motora. Nos resultados, os idosos sedentários apresentaram graus médios mais elevados sem todas as dimensões da depressão comparados com os praticantes de exercícios físicos regulares. Na discussão, são abordadas as hipóteses neurofisiológicas e psicossociais para explicar os efeitos positivos do exercício regular sobre o humor dos praticantes.
\end{abstract}

Palavras-chave: exercício físico, depressão, idosos, sedentarismo.

\begin{abstract}
The number of people over 60 years of age in the Brazilian population is around $12.5 \%$ of the total. Aerobic capacity, muscle strength, flexibility, balance, time of reaction and movement, agility and coordination are reduced in old age. This can entail numerous difficulties in carrying out the daily tasks and the functional independence of the elderly. They often feel useless, anxious, frustrated, moody, angry, and depressed. On the other hand, the practice of regular physical activities can bring improvements to their physical and mental health. This research investigated the relationship between regular physical exercise and depression in elderly individuals. A total of 160 people from 60 to 85 years of age participated in the study, of which 84 were of different types of regular physical activity and 76 were
\end{abstract}


sedentary. Depression Scale for the Elderly was used to assess depression, which consists of cognitive-affective and somatic-motor dimensions. The Student's t test revealed that the sedentary elderly presented significantly higher average degrees in both dimensions and in total depression, compared to the elderly practicing regular physical exercises. In the discussion, we discuss the neurophysiological and psychosocial hypotheses to explain the positive effects of regular exercise on the mood of the practitioners.

Keywords: physical exercise, depression, elderly, sedentary.

\section{RESUMEN}

El número de personas con más de 60 años de edad en la población brasileña se encuentra en torno del $12,5 \%$ del total. La capacidad aeróbica, la fuerza muscular, la flexibilidad, el equilibrio, el tiempo de reacción y el movimiento, la agilidad y la coordinación se reducen en la vejez. Esto puede acarrear innumerables dificultades para la realización de las tareas cotidianas y para la independencia funcional de las personas mayores. Estos se sienten a menudo inútiles, ansiosos, frustrados, malhumorados, irritados y depresivos. Por otro lado, la práctica de actividades físicas regulares puede traer mejoras para la salud física y mental de los mismos. Esta investigación investigó la relación entre el ejercicio regular y la depresión en los ancianos. Participó en la investigación 160 personas de 60 a 85 años de edad, siendo 84 practicantes de tipos variados de actividades físicas regulares y 76 sedentarios. Para evaluar la depresión se utilizó Escala de Depresión para los ancianos, constituida por las dimensiones cognitiva-afectiva y somáticamotora. El test $t$ de Student reveló que los ancianos sedentarios presentaron grados medios significativamente más elevados en las dos dimensiones y en la depresión total comparados con los ancianos practicantes de ejercicios físicos regulares. En la discusión, se abordan las hipótesis neurofisiológicas y psicosociales para explicar los efectos del ejercicio regular sobre el humor de los practicantes.

Palabras clave: ejercicio físico, depresión, mayores, sedentarismo.

Com os crescentes avanços tecnológicos, culturais e médicos, é possível observar um contínuo crescimento da expectativa de vida do brasileiro, que passou, segundo o Instituto Brasileiro de Geografia e Estatística (IBGE, 2015), de 69,83 anos, em 2000, para 75,44 anos, em 2015. Essa mudança, combinada com a queda da natalidade, transformou a pirâmide etária do Brasil. O número de idosos passou a corresponder a $12,5 \%$ da população brasileira total (IBGE, 2015). Este quadro sugere novos problemas sociais, na medida em que não houve, simultaneamente, novas políticas públicas voltadas ao idoso nas mesmas proporções do crescimento desse estrato populacional (Veras \& Oliveira, 2018). O presente estudo buscou contribuir com subsídios para a prevenção e melhoria das condições de saúde da população idosa.

O envelhecimento é um processo do desenvolvimento natural de todo ser vivo. Quando se é jovem, o amadurecimento possui conotação positiva, pois há muitas perspectivas na vida, envelhecer é sinônimo de evoluir. No entanto, quando a vida já tem sua maior parte no 
passado, envelhecer pode adquirir um sentido mais negativo, visto que a cultura contemporânea tende a excluir e marginalizar o idoso, tratando-o como uma pessoa inválida. A palavra velhice pode passar, então, a significar decadência (Freitas, Queiroz, \& Sousa, 2010; Morando, Schmitt, Ferreira, \& Mármora, 2018).

Assim, neste estágio do desenvolvimento humano, com frequência, as pessoas idosas têm a tendência a sentirem-se inúteis, ansiosas, frustradas, mal-humoradas, irritadas e depressivas. Estes sentimentos são devidos às transições que experimentam, tais como a aposentadoria e a exclusão do mercado de trabalho, o afastamento dos círculos sociais, a morte de amigos e entes queridos, dentre outras, e podem levar à falta de sentido da vida (Silva, et al. 2014). Matias, Fonsêca, Gomes e Matos (2016) observaram uma relevante prevalência de indícios depressivos entre os idosos, mesmo em amostras não clínicas. Muitas vezes, o início da depressão passa despercebido ou não é diagnosticado com precisão.

Concomitante, na dimensão física, em geral, a capacidade aeróbica, a força muscular, a flexibilidade, o equilíbrio, o tempo de reação e movimento, a agilidade e a coordenação são reduzidas na velhice (Fechine \& Trompieri, 2012). Isso tudo pode acarretar inúmeras dificuldades para a realização das atividades cotidianas e para a independência funcional. Além disso, as facilidades da vida moderna podem acarretar mais consequências negativas para a terceira idade. O idoso pode render-se à vida facilitada pela tecnologia, tornando-se sedentário, por exemplo, passando muitas horas seguidas sentado assistindo televisão. Este fenômeno pode contribuir para limitar sua autonomia e reduzir sua capacidade funcional, que já são naturalmente menores do que nos indivíduos mais jovens. Esse sedentarismo prejudica a saúde do idoso e pode, até mesmo, levar ao desenvolvimento de doenças, como a diabetes, a osteoporose e, inclusive, as doenças psicológicas como a depressão (Santos et al., 2015). Garcia et al. (2017) realizaram uma revisão sistemática sobre a inatividade física e a depressão em idosos no Brasil. Após os recortes usados para filtrar a pesquisa, selecionaram cinco artigos publicados nos últimos oito anos. Todos os estudos selecionados apontaram para uma associação inversa entre atividade física e os sintomas depressivos.

Segundo a American Psychiatric Association (2014), as principais características da depressão são humor triste, vazio ou irritável seguido de alterações cognitivas e somáticas que afetam o funcionamento normal das pessoas de forma recorrente. No diagnóstico, é preciso discriminar a tristeza e luto normais do transtorno depressivo. Uma pessoa em depressão precisa apresentar cinco dos nove sintomas listados, sendo que o primeiro ou o segundo devem estar presentes: (1) humor deprimido na maior parte de todos os dias; (2) interesse ou prazer nitidamente diminuído em relação a 
quase todas as atividades diárias; (3) perda ou ganho significativos de peso; (4) insônia ou sono excessivo diariamente; (5) agitação ou lentidão psicomotora diariamente; (6) fadiga ou perda de energia diariamente; (7) sentir-se sem valor ou com culpa excessiva, o tempo todo; (8) habilidade reduzida de pensar ou se concentrar, na maior parte do tempo; (9) Pensamentos recorrentes sobre: morte, suicídio sem plano, tentativa de suicídio ou plano para suicídio. Além disso, esses sintomas necessitam afetar de forma impactante 0 convívio social, o trabalho ou outras áreas importantes da vida do indivíduo.

Os idosos deprimidos tendem a ter menos sintomas afetivos e mais alterações cognitivas, somáticas e perda de interesse do que os adultos jovens deprimidos. Embora as taxas de suicídio nos idosos estejam diminuindo, elas ainda são mais altas do que em adultos jovens e estão mais associadas à depressão (Fernandes-Eloi \& Lourenço, 2019; Wang \& Blazer, 2015). A pessoa idosa pode encarar a velhice não apenas como um estado de perda, mas pode transformá-lo a partir das práticas de comportamentos positivos. Desta forma, mostra-se importante a introdução da atividade ou exercício físico regular em sua rotina, para trazer melhorias não apenas para a saúde física, mas também para a sua saúde mental (Santos \& Souza, 2015).

A revisão de literatura realizada por Catalan-Matamoros, GomezConesa, Stubbs e Vancampfort (2016) indicou que o exercício físico é seguro e eficaz na redução dos sintomas depressivos em pessoas idosas. Como o exercício físico tem muitos outros benefícios à saúde, deve ser considerado como uma intervenção central no tratamento multidisciplinar de idosos que sofrem de depressão.

Muitos estudos têm produzido evidências empíricas acerca das relações entre o exercício físico e a depressão em idosos. Branco et al. (2015) examinaram os níveis de depressão de idosos antes e após a participação dos mesmos no Programa Nacional de Marcha e Corrida de Portugal com intervalo de seis meses. Os sintomas depressivos apresentaram uma melhora significativa do pré para o pós-teste. Ferreira, Roncada, Tiggemann e Dias (2014) avaliaram os níveis de depressão de idosos praticantes de diversos tipos de exercícios físicos e concluíram que essas práticas reduziram os níveis de depressão. Bhamani, Khan, Karim e Mir (2015) encontraram que, quanto maior o tempo dispendido às atividades físicas pelos idosos paquistaneses, menores os graus de depressão. No estudo de Melo et al. (2017) também o tempo de prática regular de dança apresentou uma associação negativa e significativa com a depressão.

Para a depressão leve e moderada, o efeito do exercício pode ser comparável com a medicação antidepressiva e a psicoterapia; para depressão grave, o exercício parece ser uma valiosa terapia complementar aos tratamentos tradicionais (Knapen, Vancampfort, 
Morie, \& Marchal, 2015). Melo et al. (2014) realizaram um experimento com idosos diagnosticados com Transtorno de Depressão Maior, os quais foram submetidos a exercícios aeróbicos, de força e generalizados de baixa intensidade. Os grupos de exercícios aeróbicos e de força apresentaram redução significativa nos sintomas da depressão após três meses de intervenção. Essas alterações podem ser devidas aos aspectos fisiológicos gerados pelo exercício e aos fatores psicossociais.

É provável que os efeitos redutores da atividade física sobre a depressão não possam ser explicados por um único mecanismo atuando de forma isolada. Em vez disso, os efeitos são, provavelmente, devidos à contribuição de vários mecanismos que se manifestam em níveis neurofisiológicos (por exemplo, neurogênese hipocampal, regulação do eixo suprarrenal hipotálamo-hipófise) e psicológicos (por exemplo, humor, sentimentos de domínio, autoeficácia), conforme Faulkner e Carless (2006).

Essas duas hipóteses principais tentam explicar a ação do exercício físico sobre a melhoria na função cognitiva dos idosos. Na perspectiva fisiológica, os efeitos seriam explicados por: aumento no transporte de oxigênio para o cérebro, síntese e degradação de neurotransmissores, liberação de serotonina e diminuição da viscosidade sanguínea. Na perspectiva psicológica, os efeitos seriam decorrentes da diminuição da ansiedade, melhora na autoestima e na cognição, além da redução do estresse. O exercício vigoroso é fator preponderante à redução da tensão. Os exercícios físicos também trazem benefícios aos distúrbios do sono, aos transtornos de humor e aos aspectos cognitivos como memória e aprendizagem (Batista \& Oliveira, 2015; Hua, Santos-Galduroz, Aman, Bagesteiro, \& Safons, 2018).

O estudo de Mendes, Carvalho, Silva e Almeida (2017), revisão integrativa da literatura sobre o exercício físico e a depressão em idoso, abrangendo o período de 2010 a 2016 encontrou que, em geral, grupos de idosos ativos, quando comparados com grupos de sedentários, apresentam menores níveis de depressão. Os 21 estudos selecionados foram classificados em três categorias, conforme 0 conteúdo dos mesmos: (1) relação entre atividade física e depressão em idosos, em geral; (2) fatores biológicos da atividade física e depressão em idosos; (3) atividade física em grupo e a depressão em idosos. Na relação geral entre atividade física e depressão, os resultados revelaram que os indivíduos mais ativos fisicamente apresentaram menores probabilidades de desenvolverem doenças e comprometerem a qualidade do envelhecimento. Na perspectiva biológica do exercício físico, a depressão tem sido associada a alterações nos níveis de cortisol e de hidroepiandrosterona em idosos. Nos estudos revisados, apareceram evidências de que a prática de exercícios físicos regulares modula a relação entre depressão e os 
níveis de cortisol. A explicação para a associação metabólica da depressão em idosos estaria relacionada com a liberação de hormônios como a epinefrina, norepinefrina, somatotrofina, endorfina e cortisol. Também se verificaram alterações no fluxo sanguíneo e no metabolismo de várias partes do cérebro correspondentes à atenção, psicomotricidade, capacidade executiva e tomada de decisão, ideias tristes e ao aprendizado emocional. Esses resultados também podem ter sido atribuídos não apenas pelos benefícios fisiológicos, mas pela prática realizada em grupo, contribuindo para o desenvolvimento de habilidades relativas às relações sociais (Godoy, 2002; Owaria, Miyatakeb, \& Kataoka, 2018; Werneck, Bara Filho, \& Ribeiro, 2005).

Para investigar este problema de pesquisa no Brasil, Giavoni, Melo, Parente e Dantas (2008) construíram uma medida para avaliar a depressão na terceira idade, especialmente para ser usada como instrumento na atividade de pesquisa. Os pesquisadores elaboraram uma estrutura fatorial para o construto depressão composto por três fatores: cognitivo, humor e somático-motor. Os itens designados para a representação dos construtos foram submetidos à análise semântica e de juízes e, após, a Escala de Depressão para Idosos (EDI) foi respondida por 340 pessoas com idade média de 66,8 ( $D P=$ $4,71)$ anos. Os escores foram submetidos à Análise Fatorial Exploratória que extraiu dois fatores: cognitivo-afetivo e somáticomotor. Esses fatores apresentaram muito bons índices de consistência interna, 0,93 e 0,89 respectivamente, e também evidências de validade de convergência com o Beck Depression Inventory (Gorenstein \& Andrade, 1998).

A presente investigação, por meio de um delineamento de pesquisa entre participantes, comparou os escores de depressão de um grupo de idosos praticantes de exercícios físicos regulares com os escores de um grupo de idosos sedentários. Além disso, num delineamento dentre participantes, buscou relacionar as variáveis idade e tempo de prática do exercício com as dimensões específicas e global da depressão nos idosos ativos.

\section{Método}

\section{Participantes}

A amostra selecionada por conveniência foi constituída de 160 pessoas idosas com idades variando entre 60 e 85 anos. Os participantes que praticavam exercícios físicos regulares eram 84 $(52,5 \%)$, sendo 70 mulheres e 14 homens. A idade dos praticantes variou de 61 a 85 anos $(M=70$ anos; $D P=6$ anos). Os sedentários eram $76(47,5 \%)$, sendo 44 mulheres e 32 homens com idades entre 60 e 82 anos $(M=70,9 ; D P=6,06)$. As atividades físicas praticadas 
pelos idosos eram: ginástica $(n=30)$, dança $(n=12)$, musculação ( $n$ $=4)$, natação $(n=6)$, yoga $(n=2)$, tai chi chuan $(n=2)$, ciclismo $(n=4)$, caminhada $(n=12)$, pilates $(n=8)$ e RPG $(n=4)$. O tempo de prática dessas atividades físicas regulares variou de dois a 780 meses ( $M=7$ anos e meio; $D P=12$ anos), com uma frequência média de três vezes por semana.

\section{Instrumento}

Para avaliar a depressão foi utilizada a Escala de Depressão para Idosos (EDI) de Giavoni et al. (2008), que possui dois fatores: o Cognitivo-Afetivo (27 itens) e o Somático-Motor (11 itens), totalizando 38 itens. O fator Cognitivo-Afetivo compreende os sintomas cognitivos e afetivos, tais como: pessimismo recorrente, baixa motivação, baixa autoestima, culpa, sensação de fracasso, perda da concentração e/ou memória, tristeza, rejeição, apatia, medo, irritabilidade e ansiedade, entre outros. O fator SomáticoMotor compreende a parte físico/motora da depressão, o que inclui sintomas de retardo ou agitação psicomotora, alterações no sono e no apetite, perda de libido e imunossupressão. Segundo Giavoni et al. (2008), além dos dois fatores específicos, a EDI também forneceria uma medida geral de depressão, ou seja, teria um Fator de Depressão Geral. Giavoni et al. (2008) mostraram evidências de validade fatorial e consistência interna para os dois fatores e fator geral (alfas de Cronbach variaram de 0,95 a 0,89) e evidências de validade convergente entre a EDI e o Beck Depression Inventory (BDI) adaptado por Gorestein e Andrade (1998). A EDI é respondida por meio de uma escala do tipo Likert de cinco pontos: (1) "Não concordo nada"; (2) "Concordo um pouco"; (3) "Concordo mais ou menos"; (4) "Concordo bastante"; (5) "Concordo totalmente". Os autores da EDI concluíram que o instrumento está habilitado para a pesquisa com idosos na área da atividade física. A normatização do mesmo dependerá de estudos futuros que verifiquem as validades concorrente e de critério preditivo, para que possa ser utilizado com fins diagnósticos.

\section{Procedimentos de Coleta de Dados}

O projeto foi submetido e aprovado pela Comissão de Ética em Pesquisa da instituição a que se encontra vinculado por meio do parecer no 730.038. Todos participantes desta pesquisa foram informados do objetivo da pesquisa e que as identidades dos mesmos seriam mantidas em sigilo. Após estes esclarecimentos, foi solicitada aos participantes a leitura do Termo de Consentimento Livre e Esclarecido e, caso houvesse concordância com os termos do mesmo, foi pedido para que assinassem o documento. Os idosos praticantes 
de exercício regular foram abordados individualmente e responderam aos questionários em Academias da Terceira Idade. Já os idosos sedentários responderam em grupo, numa Universidade da Terceira Idade, todas localizadas na cidade do Rio de Janeiro/RJ.

\section{Procedimentos de Análise de Dados}

Os dados foram digitados no Statistical Package of Social Sciences (SPSS), versão 23 e analisados por meio de estatísticas descritivas e Teste $t$ de Student para amostras independentes, comparando os escores de depressão entre idosos praticantes de atividades físicas e idosos sedentários. Também foi utilizado o Coeficiente de Correlação de $r$ de Pearson para avaliar as relações entre as variáveis Idade dos participantes, Tempo de prática do exercício e as dimensões da EDI no grupo de idosos ativos.

\section{Resultados}

A análise descritiva dos escores dos participantes nas variáveis observadas revelou a anormalidade dos mesmos, conforme os testes univariados Kolmogorov-Smirnov e Shapiro-Wilk. No entanto, os valores de assimetria e curtose para o fator Cognitivo-Afetivo foram 1,629 e 2,329, para o Somático-Motor, 1,136 e 0,403, e para a EDI total, 1,220 e 0,617 , respectivamente, o que não caracteriza numa violação extrema da normalidade, $< \pm 2$ e a curtose $< \pm 5$ (Finney \& DiStefano, 2006). O Teste de Levene para igualdade de variâncias revelou que as amostras não apresentavam homogeneidade das mesmas, que é um pressuposto do Teste $t$. No entanto, quando as variâncias das amostras são desiguais, o SPSS, atualmente, tem um método de cálculo do valor $t$ que permite superar esta restrição e realizar o teste.

Tabela 1

Estatísticas Descritivas, Teste $t$ de Student para Idosos Praticantes e Não Praticantes de Exercícios Físicos Regulares, Intervalos de Confiança e Tamanhos dos Efeitos

\begin{tabular}{|c|c|c|c|c|c|c|c|c|c|}
\hline \multirow{3}{*}{$\begin{array}{l}\text { Cognitivo- } \\
\text { Afetivo }\end{array}$} & & $n$ & $M$ & $D P$ & $t$ & $g l$ & Dif $M$ & IC $95 \%$ da Dif & $d$ \\
\hline & Não & 76 & 1,7 & 0,65 & 3.796 & 158 & 0,479 & $0,227-0,731$ & 0,87 \\
\hline & Sim & 84 & 1,3 & 0,44 & & & & & \\
\hline \multirow{2}{*}{$\begin{array}{l}\text { Somático- } \\
\text { Motor }\end{array}$} & Não & 76 & 2,2 & 0,86 & 3.517 & 158 & 0,620 & $0,268-0,971$ & 0,79 \\
\hline & Sim & 84 & 1,6 & 0,70 & & & & & \\
\hline EDI & $\begin{array}{l}\text { Não } \\
\text { Sim }\end{array}$ & $\begin{array}{l}76 \\
84\end{array}$ & $\begin{array}{l}2,0 \\
1,4\end{array}$ & $\begin{array}{l}0,73 \\
0,54\end{array}$ & 3.798 & 158 & 0,549 & $0,261-0,838$ & 0,86 \\
\hline
\end{tabular}

Nota. Em todas as comparações o nível de significância foi de $p<0,001$. 
O Teste $t$ de Student para amostras independentes mostrou diferenças estatísticas significativas entre as médias de depressão dos idosos praticantes de exercícios físicos e dos sedentários. Nos fatores Cognitivo-Afetivo e na EDI total, os níveis médios dos sedentários em depressão foram superiores aos dos praticantes de exercícios físicos regulares (Tabela 1 e Figura 1). Para todas as diferenças encontradas, os tamanhos dos efeitos $(d)$ foram considerados grandes de acordo com classificação de Cohen (1988). O $d$ é um valor padronizado, ou seja, um escore padrão. Neste estudo, em geral, todos os valores $d$ calculados ficaram em torno de 0,80 , o que significa, em todas as comparações, que as médias se distanciaram umas das outras o equivalente a quase um desvio padrão.

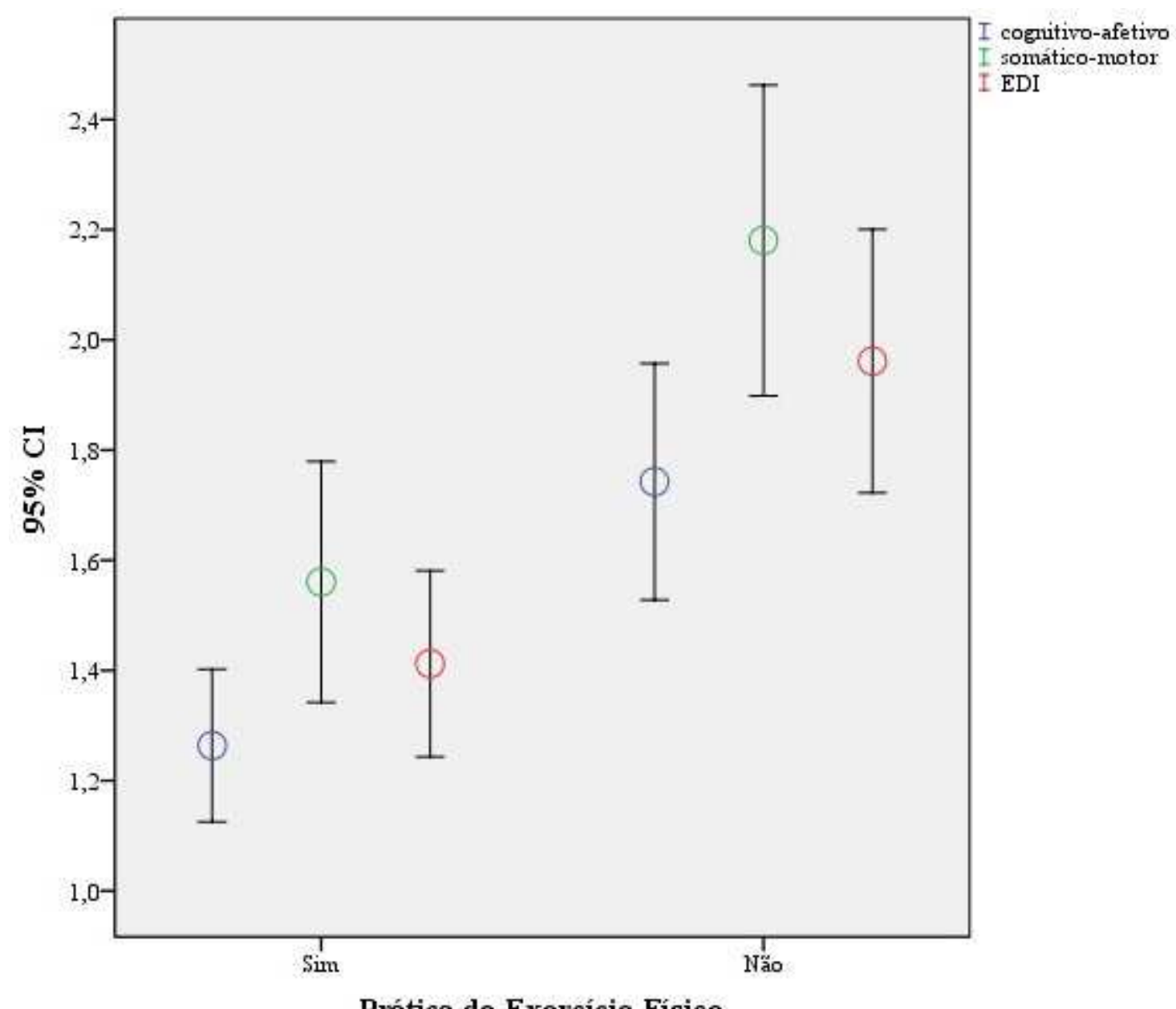

Figura 1

Gráficos de barra de erros dos fatores Cognitivo-Afetivo, Somático-Motor e EDI total para os grupos praticantes e não praticantes de exercícios físicos regulares.

O Coeficiente de Correlação de Pearson não apurou relações estatísticas significativas $(p<0,05)$ entre a Idade dos participantes, 0 Tempo de Prática de atividade física e os fatores Cognitivo-Afetivo, 
Somático-Motor e a EDI total para os idosos praticantes de atividades físicas regulares. O grupo de praticantes com maior Tempo de Prática ( $\geq 100$ meses) também não revelou relações significativas entre essas mesmas variáveis (Tabela 2 ).

Tabela 2

Coeficientes de Correlação de Pearson entre Idade, Tempo de Prática dos Participantes e os Escores nas Dimensões da EDI para a Amostra Total e para o Grupo com Maior Tempo de Prática

\begin{tabular}{llcc}
\hline & & Idade & Tempo de Prática \\
\hline \multirow{3}{*}{ Amostra total } & Cognitivo-Afetivo & $-0,28$ & $-0,15$ \\
& Somático-Motor & $-0,10$ & $-0,19$ \\
& EDI & $-0,18$ & $-0,18$ \\
\cline { 2 - 4 }$\geq 100$ meses & Cognitivo-Afetivo & - & $-0,26$ \\
& Somático-Motor & - & $-0,25$ \\
& EDI & - & $-0,26$ \\
\hline
\end{tabular}

Nota. Todas as correlações apresentaram valor-p>0,05.

\section{Discussão}

Os resultados do presente estudo demonstraram que o exercício físico parece estar associado à significativa redução nos índices de depressão em idosos. Várias pesquisas nacionais e internacionais têm encontrado resultados semelhantes. Na pesquisa atual, a média de indicativos de sintomas cognitivos da depressão no grupo de idosos ativos foi significativamente menor do que no grupo de idosos sedentários. Almeida, Mourão e Coelho (2018) e Hua et al. (2018) também encontraram resultados que corroboram que a prática regular de atividade física apresentou efeito significativo sobre a redução da depressão e, concomitantemente, na preservação das funções cognitivas dos mesmos.

No processo do desenvolvimento anatomofisiológico no envelhecimento, o conhecimento do declínio da estrutura do sistema nervoso central é importante para as intervenções que visam a promoção, a prevenção e a recuperação das funções e da saúde (Scianni, Faria, Silva, Benfica, \& Faria, 2019). Conforme Sugiura (2016), com o aumento da idade, há a tendência de agravar-se a atrofia nas regiões do hipocampo, córtex frontal, parietal e temporal devido à perda de tecidos e alterações na mielinização dos nervos e, consequentemente, redução da substância branca. Estas regiões são responsáveis por funções, tais como: memória, motricidade, planejamento motor e associação de informações.

O exercício físico pode agir como um fator de proteção e retardamento dessa atrofia no sistema nervoso central, aumentando a sobrevida do mesmo, a vascularização, a formação e estimulação neuronal, assegurando um aumento dos neurotransmissores 
(serotonina, dopamina e noraepinefrina) na corrente sanguínea, que são bastante reduzidos durante a depressão. Essas mudanças neurológicas positivas podem contribuir para o desenvolvimento das habilidades para lidar com tensões e frustrações e da autoestima (Godoy, 2002; Nóbrega et al., 1999; Werneck et al., 2005).

Os resultados desta pesquisa atual também revelaram que os escores médios dos idosos ativos na dimensão Somato-Motora da depressão foram menores do que os escores médios dos idosos sedentários. Isto pode ser explicado por outro benefício da prática do exercício físico na vida do idoso, a conquista da sua independência e autonomia. Haverá, possivelmente, na saúde física, um aumento da capacidade de bombeamento do coração, da força de músculos respiratórios, da massa muscular, da densidade óssea e da resistência dos tendões e ligamentos. Esses fatores contribuem com uma redução do risco de quedas e fraturas (Bento \& Sousa, 2017) e, ainda, aumentam a independência da pessoa idosa para a realização de simples atividades da rotina (Rodrigues, Leitão, Cavalcante, \& Aragão, 2016) como o saltar num ponto de ônibus, subir um lance de escada e ir às compras na padaria ou no mercado próximo andando (Nascimento et al., 2015). Estas habilidades contribuiriam também para o aumento da autoconfiança e da autoestima do idoso e, consequentemente, para a prevenção ou redução da depressão.

$\mathrm{Na}$ perspectiva psicológica, algumas hipóteses tentam explicar a influência do exercício físico sobre a depressão nos idosos. A Hipótese Cognitivo-Comportamental, inspirada no modelo de depressão de Beck, postula a presença automática de pensamentos negativos, de erros lógicos sistemáticos, ou seja, o esquema depressogênico. 0 exercício físico, por meio da substituição dos sentimentos e pensamentos negativos por sentimentos e pensamentos positivos, poderia romper com a chamada espiral depressiva. O sentimento de prazer proveniente da interação e do reforço social compartilhado com o grupo no ambiente do exercício físico é postulado pela Hipótese da Interação Social. A Hipótese da Distração de Sachs sustenta que a prática do exercício físico proporcionaria uma distração das preocupações do cotidiano (Gabriel \& Pozzobon, 2013; Godoy, 2002).

Apesar disso, ainda há muitas medidas a serem tomadas para retirar o idoso da posição estigmatizada em que se encontra (Morando et al., 2018). É importante ressaltar que o estudo aqui apresentado pretende não apenas servir de apoio a pesquisas mais avançadas na área da saúde geriátrica, mas também como uma forma de chamar a atenção para formas simples e econômicas de melhorar a saúde desta parcela da população brasileira, que é cada vez mais numerosa e, contudo, é tão negligenciada pelas políticas públicas. A utilização do exercício físico como tratamento complementar para a depressão foi evidenciada na metanálise de Schuch et al. (2016), que 
encontraram efeitos maiores para intervenções em Transtorno de Depressão Maior utilizando exercícios aeróbicos intensos e moderados. Esses dados apoiaram fortemente a posição de que o exercício supervisionado é um tratamento complementar baseado em evidências para a depressão.

Por fim, esta pesquisa mostrou-se bem-sucedida, cobrindo a hipótese feita inicialmente. Evidenciou-se uma diferença expressiva entre os participantes que praticam atividade física e aqueles que não praticam atividade física no que tange aos índices de depressão analisados e representados pelos fortes tamanhos de efeitos calculados. Não há dúvidas acerca da relação entre o exercício físico e a depressão em idosos, haja vista que está forte e claramente relatada na literatura dessa linha de pesquisa, tanto no âmbito nacional quanto internacional, conforme revelaram várias revisões sistemáticas e metanálises abordadas na revisão deste estudo (Garcia et al., 2017; Mendes et al., 2017; Schuch et al., 2016).

Não foram encontradas correlações significativas entre a idade e o tempo de prática de exercícios físicos dos participantes idosos e a depressão no grupo de praticantes. Melo et al. (2017) também não encontraram relação significativa entre o tempo de prática da dança e a depressão em praticantes com menos tempo nesta atividade, mas sim entre os que apresentavam mais de 100 meses de prática da dança, que é uma atividade física de intensidade moderada. $\mathrm{Na}$ pesquisa atual, também não foi encontrada correlação significativa entre essas variáveis nos praticantes com mais tempo de prática de exercícios físicos. A literatura indica que é importante considerar também a intensidade, a duração e a frequência do exercício físico sobre a depressão em idosos (Schuch, 2016). Por exemplo, Chang, $\mathrm{Lu}, \mathrm{Hu}, \mathrm{Wu}$ e Hu (2017) encontraram que exercícios físicos com pelo menos 15 minutos de duração, três vezes por semana e de intensidades moderadas foram significativamente associados com baixo risco de sintomas depressivos. No presente estudo, dada as características da amostra não foi possível compor grupos de praticantes de acordo com a intensidade, duração e frequência das atividades físicas.

Evidente que este estudo apresenta limitações. Sugere-se que novas investidas possam contar com amostras maiores, equilibradas entre os sexos masculino e feminino e representativas das diversas regiões brasileiras. Além disso, sugere-se que, nessas amostras, possam ser contemplados os praticantes de exercícios físicos de diferentes intensidades, durações e frequências. Destarte os limites do estudo atual, o efeito identificado entre as variáveis investigadas mostrou-se muito forte, os dados obtidos corroboraram a expectativa inicial e apontaram para a importância da atividade física como fator que pode interferir ativa e positivamente na promoção da saúde mental das pessoas e, nesse caso, a população de pessoas idosas. 


\section{Referências}

Almeida, E., Mourão, I., \& Coelho, E. (2018). Saúde mental em idosos brasileiros: Efeito de diferentes programas de atividade física. Psicologia, Saúde \& Doenças, 19(2), 390-404. doi:10.15309/18psd190218

American Psychiatric Association. (2014). DSM-5: Manual Diagnóstico e Estatístico de Transtornos Mentais. Porto Alegre: Artmed.

Batista, J. I., \& de Oliveira, A. (2015). Efeitos psicofisiológicos do exercício físico em pacientes com transtornos de ansiedade e depressão. Revista Corpoconsciência, 19(3), 1-10. Recuperado de

http://periodicoscientificos.ufmt.br/ojs/index.php/corpoconscien cia/article/view/3974

Bento, J. R., \& Sousa, N. D. (2017). Exercício físico na prevenção de quedas do idoso da comunidade: Revisão baseada na evidência. Revista Brasileira de Medicina Família e Comunidade, 12(39), 117. doi:10.5712/rbmfc12(39) 1658

Bhamani, M. A., Khan, M. M., Karim, M. S., \& Mir, M. U. (2015). Depression and its association with functional status and physical activity in the elderly in Karachi, Pakistan. Asian Journal of Psychiatry, 14, 46-51. doi:10.1016/j.ajp.2014.12.004

Branco, J. C., Jansen, K., Teixeira Sobrinho, J., Carrapatoso, S., Spessato, B., Carvalho, J., ... Silva, R. A. (2015). Physical benefits and reduction of depressive symptoms among the elderly: Results from the Portuguese "National Walking Program". Ciência \& saúde coletiva, 20(3), 789-795. doi:10.1590/1413-81232015203.09882014

Catalan-Matamoros, D., Gomez-Conesa, A., Stubbs, B., \& Vancampfort, D. (2016). Exercise improves depressive symptoms in older adults: An umbrella review of systematic reviews and meta-analyses. Psychiatry Research, (244), 202209. doi:10.1016/j.psychres.2016.07.028

Chang, Y.-C., Lu, M.-C., Hu, I-H., Wu, W.-C., Hu, S. C. (2017). Effects of different amounts of exercise on preventing depressive symptoms in community-dwelling older adults: A prospective cohort study in Taiwan. BMJ Open, 7(4), 1-10. doi:10.1136/bmjopen-2016-014256

Cohen, J. (1988). Statistical power analysis for the behavioral sciences (2a ed.). Hillsdale: Lawrence Erlbaum Associates.

Faulkner, G., \& Carless, D. (2006). Atividade física no processo de reabilitação psiquiátrica: Questões teóricas e metodológicas. Revista de Reabilitação Psiquiátrica, 29(4), 258-266. doi: 10.2975 / 29.2006.258.266 
Fernandes-Eloi, J., \& Lourenço, J. R. C. (2019). Suicídio na Velhice: Um estudo de revisão integrativa da literatura. Revista CES Psicología, 12(1), 80-95. doi:10.21615/cesp.12.1.7

Ferreira, L., Roncada, C., Tiggemann, C. L., \& Dias, C. P. (2014). Avaliação dos níveis de depressão em idosos praticantes de diferentes exercícios físicos. ConScientia e Saúde, 13(3), 405410. doi: $10.5585 /$ ConsSaude.v13n3.4839

Fechine, B. R. A., \& Trompieri, N. (2012). O Processo de Envelhecimento: As principais alterações que acontecem com o idoso com o passar dos anos. Revista Científica Internacional, 1(7), 106-132. doi: $10.6020 / 1679-9844 / 2007$

Finney, S. J., \& DiStefano, C. (2006). Non-normal and categorical data in structural equation modeling. In G. R. Hancock, \& R. O. Mueller (Eds.), Structural equation modeling: A second course (pp. 269-314). Greenwich, CT: Information Age Publishing.

Freitas, M. C. de, Queiroz, T. A., \& Sousa, J. A. V. (2010). O significado da velhice e da experiência de envelhecer para os idosos. Revista da Escola de Enfermagem da USP, 44(2), 40712. doi: $10.1590 / S 0080-62342010000200024$

Gabriel, K., \& Pozzobon, A. (2013). Efeito da atividade física na depressão e na qualidade de vida de idosos. Cinergis, 14(2), 134-137. doi:10.17058/cinergis.v14i2.3643

Garcia, L. A. A., Milani, J., Celeste, L. F. N., Chagas, L. M. O., Caixeta, T. P., \& Santos, A. S. (2017). Inatividade física e depressão em idosos no Brasil: Uma revisão sistemática. Revista Família, Ciclos de Vida e Saúde no Contexto Social, 5(1), 66-74. doi:10.18554/refacs.v5i1.1916

Giavoni, A., Melo, G. F., Parente, I., \& Dantas, G. (2008). Elaboração e validação da Escala de depressão para idosos. Cadernos de Saúde Pública, 24(5), 975-982. doi:10.1590/S0102$311 \times 2008000500004$

Godoy, R. F. (2002). Benefícios do exercício físico sobre a área emocional. Revista Movimento, 8(2), 7-15. doi:10.22456/19828918.2639

Gorenstein, C., \& Andrade, L. (1998). Inventário de depressão de Beck: Propriedades psicométricas da versão em português. Revista de Psiquiatria Clínica, 25(5), 245-250.

Hua, F. Y., Santos-Galduroz, R. F., Aman, F. C. S., Bagesteiro, L. B., \& Safons, M. P. (2018). Influências da atividade física na cognição e na depressão durante o envelhecimento. Arquivos Brasileiros de Educação Física, 1(1), 157-166. doi: 10.20873/abef.v1i1.6179

Instituto Brasileiro de Geografia e Estatística. (2015). População: Projeção da população do Brasil e das Unidades da Federação. Recuperado http://www.ibge.gov.br/apps/populacao/projecao/ 
Knapen, J., Vancampfort, D., Morie, Y., \& Marchal, Y. (2015). Exercise therapy improves both mental and physical health in patients with major depression. Disability and Rehabilitation: An International Multidisciplinary Journal, 37(16), 1490-1495. doi:10.3109/09638288.2014.972579

Lee, H., Lee, J.-A., Brar, J. S., Rush, E. B., \& Jolley, C. J. (2014). Physical activity and depressive symptoms in older adults. Geriatric Nursing, 35(1), 37-41. doi:10.1016/j.gerinurse.2013.09.005

Matias, A. G. C., Fonsêca, M. A., Gomes, M. L. F., \& Matos, M. A. A. (2016). Indicadores de depressão em idosos e os diferentes métodos de rastreamento. Einstein, 14(1), 6-11. doi: 10.1590/S1679-45082016A03447

Melo, B., Moraes, H. S., Silveira, H., Oliveira1, N., Deslandes, A. C., \& Laks, J. (2014). Efeito do treinamento físico na qualidade de vida em idosos com depressão maior. Revista Brasileira de Atividade Física e Saúde, 19(2), 205-214. doi: 10.12820/rbafs.v.19n2p205

Melo, C. C., Costa, V. T., Boletini, T. L., Freitas, V. G., Costa, I. T., Arreguy, A. V., \& Noce, F. (2017). A Influência do tempo de prática de dança de salão nos níveis de depressão de idosos. Revista de Psicología del Deporte / Journal of Sport Psychology, 27(1), 67-73. https://www.rpd-online.com/article/view/v27-s1carvalho-de-melo-etal

Mendes, G. A. B., Carvalho, M. V., Silva, A. M. T. C., \& Almeida, R. J. (2017). Relação entre atividade física e depressão em idosos: Uma revisão integrativa. Revista de Atenção à Saúde, 15(53), 110-116. doi:10.13037/ras.vol15n53.4524

Morando, E. M. G., Schmitt, J. C., Ferreira, M. E. C., \& Mármora, C. H. C. (2018). O conceito de estigma de goffman aplicado à velhice. International Journal of Developmental and Educational Psychology / INFAD: Revista de Psicología, 1(2), 21-32. doi:10.17060/ijodaep.2018.n2.v1.1341

Nascimento, C. P., Lago, L. S., Almeida, R. F. F., Gusmão, M. F. S., Duarte, S. F. P., \& Reis, L. A. (2015). Desempenho motor em idosos participantes de grupos de convivência. Revista Pesquisa em Fisioterapia, 5(2), 93-101. doi:10.17267/22382704rpf.v5i2.645

Nóbrega, A. C. L., Freitas, E. V., Oliveira, M. A. B., Leitão, M. B., Lazzoli, J. K., Nahas, R. M., ... De Rose, E. H. (1999). Posicionamento oficial da Sociedade Brasileira de Medicina do Esporte e da Sociedade Brasileira de Geriatria e Gerontologia: Atividade física e saúde no idoso. Revista Brasileira de Medicina do Esporte, 5(6), 207-211. doi:10.1590/S151786921999000600002 
Owaria, Y., Miyatakeb, N., \& Kataoka, H. (2018). Relationship between social participation, physical activity and psychological distress in apparently healthy elderly people: A pilot study. Acta Medica Okayama, 72(1), 31-37. doi:10.18926/AMO/55660

Rodrigues, A. R. M., Leitão, N. M. A., Cavalcante, A. E. S., \& Aragão, M. M. (2016). Autonomia nas atividades de vida diária: Avaliação de idosos praticantes de exercícios físicos. Revista Kairós Gerontologia, 19(2), 279-293. Recuperado de https://revistas.pucsp.br/kairos/article/view/33027/22768

Santos, R. G., Medeiros, J. C., Schmitt, B. D., Meneguci, J., Santos, D. A. T., Damião, R., ... Virtuoso Júnior, J. S. (2015). Comportamento Sedentário em Idosos: Uma revisão sistemática. Motricidade, 11(3), 171-186. doi: $10.6063 /$ motricidade.3184

Santos, S. T., \& Souza, L. V. (2015). Envelhecimento positivo como construção social: Práticas discursivas de homens com mais de sessenta anos. Revista da SPAGESP, 16(2), 46-58. Recuperado de

http://pepsic.bvsalud.org/scielo.php?script=sci_arttext\&pid=S1 677-29702015000200005\&lng=pt\&tIng =pt

Schuch, F. B., Vancampfort, D., Richards, J., Rosenbaum, S., Ward, P. B., \& Stubbs, B. (2016). Exercise as a treatment for depression: A meta-analysis adjusting for publication bias. Journal of Psychiatric Research, 77, 42-51. doi:10.1016/j.jpsychires.2016.02.023

Scianni, A. A., Faria, G. S., Silva, J. S., Benfica, P. A., \& Faria, C. D. C. M. (2019). Efeitos do exercício físico no sistema nervoso do indivíduo idoso e suas consequências funcionais. Revista Brasileira de Ciências do Esporte, 41(1), 81-95. doi:10.1016/j.rbce.2018.03.026

Silva, L. M., Moreira, M. A. S. P., Bezerra, V. P., Almeida, S. A., Brenna, S. \&, Silva, A. O. (2014). Representações sociais sobre solidão por idosos institucionalizados. Journal of Research Fundamental Care Online, 6(supl.), 1-9. doi:10.9789/21755361.2014.v6i5.1-9

Sugiura, M. (2016). Functional neuroimaging of normal aging: Declining brain, adapting brain. Ageing Research Reviews, 30, 61-72. doi:10.1016/j.arr.2016.02.006

Wang, S., \& Blazer, D. G. (2015). Depression and Cognition in the Elderly. Annual Review Clinical Psychology, 11, 331-360. doi:10.1146/annurev-clinpsy-032814-112828

Wassink-Vossen, S., Collard, R. M., Voshaar, R. C. O., Comijs, H. C., Vocht, H. M., \& Naarding, P. (2014). Physical (in)activity and depression in older people. Journal of Affective Disorders, 161, 65-72. doi:10.1016/j.jad.2014.03.001 
Veras, R. P., \& Oliveira, M. (2018). Envelhecer no Brasil: A construção de um modelo de cuidado. Ciências \& saúde coletiva, 23(6), 1929-1936. doi:10.1590/141381232018236.04722018

Werneck, F. Z., Bara Filho, M. G., \& Ribeiro, L. C. S. (2005). Mecanismos de melhoria do humor após o exercício: Revisitando a hipótese das endorfinas. Revista Brasileira de Ciência e Movimento, 13(2), 135-144. doi: $10.18511 /$ rbcm.v13i2.634

\section{Endereço para correspondência \\ José Augusto Evangelho Hernandez}

Rua São Francisco Xavier, 524, $10^{\circ}$ andar, sala 10028D, Maracanã, CEP 20550900, Rio de Janeiro - RJ, Brasil

Endereço eletrônico: hernandez.uerj@gmail.com

\section{Rogério da Cunha Voser}

Rua Felizardo, 750, Jardim Botânico, CEP 90690-200, Porto Alegre - RS, Brasil

Endereço eletrônico: rogerio.voser@ufrgs.br

Recebido em: 16/05/2019

Reformulado em: 06/10/2019

Aceito em: 09/10/2019

\section{Notas}

* Doutor em Psicologia do Desenvolvimento pela Universidade Federal do Rio Grande do Sul e docente do Programa de Pós-Graduação em Psicologia Social da UERJ.

** Doutor em Ciências da Saúde pela Pontifícia Universidade Católica do Rio Grande do Sul e docente do Programa de Pós-Graduação em Ciências do Movimento Humano da UFRGS.

Este artigo de revista Estudos e Pesquisas em Psicologia é licenciado sob uma Licença Creative Commons Atribuição-Não Comercial 3.0 Não Adaptada. 